\title{
L'apprentissage de l'écriture scientifique au cours des formations en sciences de la santé
}

\author{
Un enjeu pour l'intégration universitaire des études de maïeutique
}

L'écriture est un mode d'expression et de communication essentiel dans le champ de la santé. Dans l'intervalle de chacun de ses congrès, notre communauté francophone en éducation médicale échange ainsi ses expériences grâce à son organe d'expression, la revue Pédagogie Médicale, média qui repose sur l'expression écrite. Par ailleurs, depuis de nombreuses années, la production d'un écrit à caractère scientifique ou professionnel, sous la forme d'un mémoire ou d'une thèse, est le sésame incontournable pour l'obtention des diplômes des filières santé, au point qu'il constitue une forme de rite initiatique. Pour autant, il n'est pas certain que la formation à l'écriture scientifique reçoive autant d'attention qu'elle le mériterait au cours de la formation des professionnels de la santé. Force est même d'admettre que son enseignement et son apprentissage restent souvent de l'ordre des codes implicites.

Il faut donc saluer la contribution originale de Marjan Nadjafizadeh et Olivier Morel, de l'Université de Lorraine, qui est proposée dans cette nouvelle livraison de la revue ${ }^{[1]}$. Ces auteurs proposent une étude qualitative qui compare les productions scientifiques d'étudiants de deux filières médicales : la maïeutique et la gynécologie obstétrique. Si les deux populations sont assez proches en termes d'appartenance disciplinaire et de recrutement à l'entrée dans les études, elles se révèlent différentes dans leur rapport aux écrits scientifiques, les futurs médecins semblant mieux réussir l'exercice que les futures sages-femmes. Les résultats de cette recherche à visée compréhensive ne sont pas généralisables sans précaution mais ils nous invitent à considérer l'utilité de l'écriture scientifique dans les formations initiales en sciences de la santé.

\section{Écrire pour communiquer et valoriser}

La fonction première des écrits scientifiques ou professionnels est de communiquer. Ils visent la valorisation et la diffusion de connaissances régulièrement actualisées en sciences de la santé. Les auteurs adressent les résultats de leurs recherches, le fruit de leur réflexion et leurs recommandations à une communauté professionnelle ciblée. Les lecteurs mettent à jour leurs connaissances, trouvent des sources d'inspiration pour explorer de nouvelles pistes de recherche, ou pour enrichir ou modifier leurs pratiques. Les uns se préparent parfois à des carrières universitaires pour lesquelles ils devront prouver un engagement important en termes d'écrits scientifiques. Les autres se présentent comme des praticiens responsables, dispensant à leurs patients des soins conformes aux données scientifiques du moment.

En toute rigueur, l'écriture scientifique se caractérise par une langue froide, neutre, dénuée d'affects. Exit les superlatifs, les phrases alambiquées ou le style ampoulé ! Le style scientifique se caractérise par des énoncés impersonnels, au point que l'on a pu définir le style scientifique idéal comme l'absence de tout style. Nul besoin donc d'être un littéraire pour 
réussir l'exercice... Cela pourrait même être considéré comme un handicap, notamment si le manuscrit devait être traduit en anglais.

\section{Écrire dès le début du travail de recherche}

L'écriture clarifie la pensée. Ecrire sa question de recherche est une étape souvent difficile. Chaque mot compte, donne du sens et peut changer l'orientation du travail qui va découler de ces quelques lignes bien rédigées : la méthode de recherche la plus appropriée, la population à étudier, les critères d'inclusion, le traitement des résultats... Si une telle étape est incontournable, elle ne représente qu'une part infime du travail de rédaction.

Bordage et Querin nous invitaient il y a quelques années, dans cette même revue ${ }^{[2]}$, à utiliser le résumé structuré comme un outil de lecture et d'évaluation, mais également de rédaction. Selon ces auteurs, rédiger le résumé structuré figurant en exergue d'une publication, ou sur la quatrième de couverture d'une thèse ou d'un mémoire, facilite la tâche de ceux qui ne savent pas par où commencer. "Très vite, les auteurs peuvent entreprendre la rédaction du corps de l'article en complétant chacune des rubriques du résumé structuré à l'aide des mots clés. Ce premier jet donnera aux auteurs une vue d'ensemble du travail à accomplir et servira de point de repère tout au long du processus de rédaction, en facilitant l'agencement et l'articulation des différentes composantes de l'article; ainsi ce jet structuré sera une source immédiate de satisfaction qui facilitera la mise en route du processus de rédaction, celui-ci pouvant être pénible au départ». Ce conseil a surement aidé beaucoup de lecteurs et il mérite d'être répété à tous les étudiants dont on dirige le travail. Finie l' angoisse de la page blanche! Le résumé structuré peut être partiellement rédigé avant même le recueil de données. Dès le choix de la thématique, le contexte peut être synthétisé, la question de recherche formulée, les objectifs précisés et les méthodes sélectionnées. Les résultats attendus seront ensuite ajustés, voire modifiés. La discussion et les conclusions viendront dans un second temps. Il suffit ensuite de dérouler chaque point pour passer du résumé à un texte plus étoffé.
Comme Marjan Nadjafizadeh et Olivier Morel le soulignent, l'écriture de recherche est bien souvent exigée trop tardivement dans le cursus et les normes d'écriture restent généralement implicites. Sans incitation particulière du directeur de mémoire, l'étudiant ne s'engagera probablement que tardivement dans la rédaction, considérant qu'elle constitue l'étape terminale de son travail. Les contraintes ne lui permettront pas de produire un texte plusieurs fois relu et parvenu à maturation. De plus, sans consigne précise donnée au départ, les exigences du jury et les critères de réussite ne sont pas clairs pour l'étudiant.

\section{Écrire pour s'intégrer à la communauté universitaire}

Dans leur article, nos collègues nancéens suggèrent que l'aisance des étudiants en médecine à écrire serait en lien direct avec le modèle de rôle des enseignants chercheurs qui encadrent leurs travaux, modèle encore peu courant dans la filière maïeutique. Cette hypothèse plaide en faveur du développement d'une culture de recherche chez les enseignants sages-femmes, au travers de leur propre parcours de formation universitaire et de leur reconnaissance en qualité d'enseignants-chercheurs.

Dans cette filière, le processus d'universitarisation (dans lequel s'engagent actuellement les filières dites paramédicales) semble dépassé depuis longtemps. C'est du moins le cas en France où, depuis 1803 (première loi sur les études médicales), une proportion significative des enseignements en école de sages-femmes est assurée par des universitaires ; le diplômé d'Etat (créé en 1917 pour remplacer le certificat d'aptitude) fut délivré par les facultés de médecine relayées il y a plusieurs décennies déjà par les universités; enfin, dès l'année 2000-2001, le recrutement à l'entrée des études devint universitaire (via le concours de fin de première année des études médicales, devenue première année commune des études de santé). Ainsi, c'est une pleine intégration que souhaitent les sages-femmes enseignants de France, concernant non seulement les études mais également la structure de formation et leurs statuts.

Pourtant, à ce jour, seules quelques écoles de sages-femmes sont intégrées à l'université. La 
première d'entre elles fut l'École universitaire de Maïeutique Marseille Méditerranée (EU3M), composante d'Aix Marseille Université depuis 2010. Les maïeuticiens enseignants de cette école ont un niveau de formation universitaire (master ou thèse). Ils ont personnellement été confrontés à une démarche de recherche scientifique valorisée par l'écriture. Le dispositif de formation en vue de la réalisation du mémoire de fin des études de sage-femme mis en place depuis 1985 est bien rodé, régulièrement ajusté. Les directions des mémoires sont confiées à des sages-femmes enseignantes, à des universitaires (médecins ou pas) et à des chercheurs, en fonction de leur domaine d'expertise dans la thématique choisie. Tous partagent la même rigueur méthodologique. La qualité des travaux s'élève progressivement, le nombre de publications aussi. Grace à un accompagnement important par l'équipe pédagogique, de plus en plus d'étudiants identifient l'utilité de leur travail autrement que pour l'obtention de leur diplôme.

\section{Quelques pistes pour favoriser et faciliter l'apprentissage de l'écriture dans les formations en sciences de la santé}

Dans beaucoup de filières de formation en santé, il est désormais demandé aux étudiants de rédiger leur travail de fin d'études selon le format d'un article à visée professionnelle ou scientifique, plutôt que sous la forme d'un mémoire ou d'une thèse. Les capacités d'analyse et de synthèse sont tout autant sollicitées. En revanche, la valorisation en est facilitée, grâce à un manuscrit déjà conforme ou presque à celui qui pourra être soumis à une revue ciblée.

L'utilisation d'un guide, tel que celui que nous avions publié dans cette revue ${ }^{[3]}$, facilite cette démarche. Selon notre expérience, en précisant les attendus institutionnels auprès des étudiants et de leurs directeurs, cet outil contribue à augmenter la qualité des travaux de fin d'études. Nous l'avons modifié au fil des ans, relevant encore le niveau de performance. Aujourd'hui nous constatons une élévation globale des mentions.

Au-delà des règles de structuration du texte, la rédaction correcte d'un texte ne s'improvise pas.
Même si des constats sont régulièrement faits d'une piètre qualité de l'écriture des articles publiés ${ }^{[4]}$, la mauvaise qualité rédactionnelle d'un manuscrit compromet ses chances de publication. Un travail de recherche bien mené peut être desservi par une expression écrite maladroite, des fautes d'orthographes ou de syntaxe. Des ateliers d'écriture peuvent ainsi être proposés dans le cadre des enseignements optionnels. Au-delà de l'apprentissage spécifique des règles propres à l'écriture scientifique, les étudiants en difficulté peuvent être en demande de réactivation des concepts clés de grammaire française, négligés depuis les années lycée au profit des apprentissages scientifiques.

L'écriture scientifique visant à rapporter des travaux originaux ne résume pas la problématique de l'écriture dans le cadre de la formation des professionnels de la santé. Il faut également mentionner, la place prépondérante de l'écriture narrative, telle qu'elle peut être développée, par exemple, dans le cadre d'un portfolio et qui poursuit d'autres finalités ${ }^{[5]}$. Dans un cas comme dans l'autre, l'écriture est évidemment bien plus qu'une activité «mécanique ». Elle soutient des processus cognitifs complexes, favorise les apprentissages en profondeur et signifiants, et promeut l'activité réflexive. À ce titre, elle occupe une place privilégiée non seulement dans le nouveau parcours de la formation à la recherche, mais plus largement dans le cadre du parcours de socialisation professionnelle. Dans la poursuite du processus d'intégration de la filière maïeutique, la formation à l'écriture des étudiants revêt des enjeux dont il faut prendre toute la mesure.

Anne DEMEESTER

Aix Marseille Université École universitaire de maïeutique Marseille Méditerranée 51 Boulevard Pierre Dramard 13344 Marseille Cedex 15, France Mailto : anne.demeester@univ-amu.fr 


\section{Références}

1. Nadjafizadeh M, Morel O. Acculturation des étudiants en filières médicale et maïeutique au genre d'écrit de recherche : le rôle différenciateur du contexte pédagogique. Pédagogie Médicale 2014;15:285-300

2. Bordage G, Querin S. Le résumé structuré : un outil de lecture, d'évaluation et de rédaction. Pédagogie Médicale 2001;2:81-4.

3. Demeester A, Cottu A-C, Balzing M-P, Chavent D, Perrin-Terrin C, Comelli C, Schonek M. Un guide pour améliorer le suivi et l'évaluation des travaux de recherche de fin d'études en sciences de la santé. Exemple d'une grille pour la validation du mémoire de fin d'études de sage-femme à Marseille. Pédagogie Médicale 2004;5:46-51.

4. Albert T. Why are medical journals so badly written? Med Educ 2004;38:6-8.

5. Levine RB, Kern DE, Wright SM. The impact of prompted narrative writing during internship on reflective practice: a qualitative study. Adv Health Sci Educ 2008;13:723-33. 